\title{
Trajectory Query Based on Trajectory Segments with Activities
}

\author{
Kaiwei Kong \\ School of Computer Science and \\ Technology, Hangzhou Dianzi \\ University \\ 151050025@hdu.edu.cn \\ Liming $\mathrm{Tu}$ \\ School of Computer Science and \\ Technology, Hangzhou Dianzi \\ University \\ tuliming@hdu.edu.cn
}

\author{
Jian Xu \\ School of Computer Science and \\ Technology, Hangzhou Dianzi \\ University \\ jian.xu@hdu.edu.cn \\ Yang $\mathrm{Wu}$ \\ School of Computer Science and \\ Technology, Hangzhou Dianzi \\ University \\ 161050040@hdu.edu.cn
}

\author{
Ming $\mathrm{Xu}$ \\ School of Computer Science and \\ Technology, Hangzhou Dianzi \\ University \\ mxu@hdu.edu.cn \\ Zhi Chen \\ School of Computer Science and \\ Technology, Hangzhou Dianzi \\ University \\ 162050110@hdu.edu.cn
}

\begin{abstract}
Searching trajectories with activities has attracted much attention in the last decade. Existing studies tend to find trajectories with activities matched to the required keywords. However, returned trajectories may have a satisfying textual matching but are spatially far from query locations. In this paper, differing with traditional work which return entire trajectories without combination,we focus on the intersecting trajectory segments and combine them into a new trajectory. A challenge of this problem is how to find qualified trajectory segments from the large search space and combine them into required trajectories. To this end, we organize trajectories into a hybrid index which enables us to utilize spatial information to prune search space efficiently. In addition, we propose a algorithm to search intersecting trajectory segments and combine them into qualified trajectories according to requirements. The effectiveness of our method is verified by empirical studies based on a real trajectory data set and a synthetic data set.
\end{abstract}

\section{CCS CONCEPTS}

- Information systems $\rightarrow$ Location based services;

\section{KEYWORDS}

activity keyword, trajectory search, trajectory segments

\section{ACM Reference Format:}

Kaiwei Kong, Jian Xu, Ming Xu, Liming Tu, Yang Wu, and Zhi Chen. 2017. Trajectory Query Based on Trajectory Segments with Activities. In Proceedings of ACM SIGSPATIAL 2017 Workshop$s$, Redondo Beach, CA, USA, November, 2017 (UrbanGIS17), 8 pages.

https://doi.org/10.1145/3152178.3152180

Permission to make digital or hard copies of part or all of this work for personal or classroom use is granted without fee provided that copies are not made or distributed for profit or commercial advantage and that copies bear this notice and the full citation on the first page. Copyrights for third-party components of this work must be honored.

For all other uses, contact the owner/author(s).

UrbanGIS17, November, 2017, Redondo Beach, CA, USA

(C) 2017 Copyright held by the owner/author(s).

ACM ISBN 123-4567-24-567/08/06 . .\$15.00

https://doi.org/10.1145/3152178.3152180

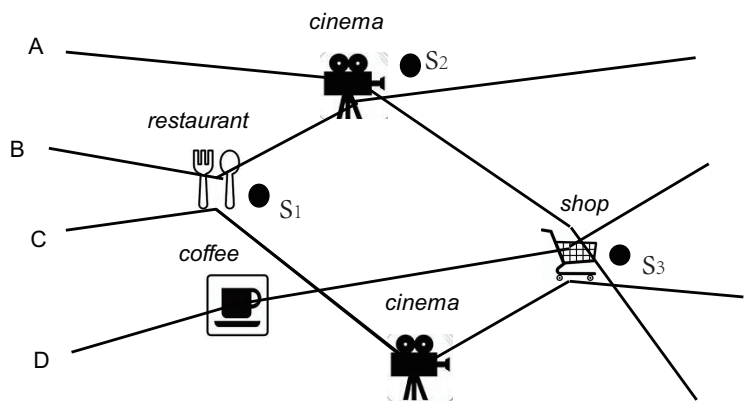

Figure 1: Trajectory query example

\section{INTRODUCTION}

Driven by the rapid development of senor technology and the Internet of Things, modern scientific community acquires more and more spatial trajectories. In recent years, many geo-related locations are tagged with a text label such as restaurant, store, hotel, place of interest. It motivates researchers to concentrate on analysis about activities on human trajectories.

To model the relationship between activity and trajectory, Zheng et al. firstly proposed the concept of activity trajectory in [17]. In activity trajectories, each geo-spatial point is associated with a set of keywords which describe activities of a user at this location. Moreover, they formulated a new activity trajectory query. Given a set of query locations associated with keywords, activity trajectory query can find activity trajectories which match to query keywords and are close to query locations in spatial.

Traditional activity trajectory search requires accurate keyword matching and searches for the top- $k$ trajectories for a user. If a user input a wrong word, traditional work may return a wrong trajectory to the user. To solve this problem, Zheng et al. propose an improved algorithm, called approximate keywords search, to search trajectories which have similar spellings of activity keywords to the query, such as "theatre" and "theater". 
However, the foregoing methods concentrate on text matching to seek qualified trajectories and ignore to improve spatial match distance so that they cannot breakthrough the limitation of spatial aspect. As exemplified in Figure 1, a user plans a series of activities near specified locations $S_{1}, S_{2}$ and $S_{3}$. Traditional activity trajectory search methods tend to return $C$ as a result because $C$ satisfies activity requirements of the user, though the cinema on $C$ is a little far from the query location $S_{2}$. Observing that $A$ and $B$ both satisfy a part of activity requirements, and they meet at a point around the query location $S_{2}$. We can combine the segment of $B$ in front of $S_{2}$ and the segment of $A$ behind to $S_{2}$ into a new trajectory as a result returned to users. The new trajectory meet user's requirement and is closer to all query locations than $C$. In order to achieve a more rational trajectory recommendation, it is essential to find combinations of qualified trajectory segments while searching the trajectory database.

In this paper, we propose a trajectory segments search approach to achieve the goal of returning more meaningful results to users. Although the process of trajectory split and combination is easy to understand, the efficiency is a more challenging problem since searching combinations of qualified trajectory segments is time consuming. To solve this problem, we plan to organize trajectories and activity points into a hybrid index. The hybrid index enables us to $\mathrm{u}-$ tilize spatial distance to prune search space efficiently. Based on this hybrid index, we develop a algorithm called Ordersensitive Activity Trajectory Segments Search (OATSS) to search combinations of trajectory segments. The algorithm starts with a search step to retrieve candidate trajectories close to query locations. Next, it searches intersection region among candidate trajectories and combine two qualified trajectory segments into a required trajectory as a part of results.

To sum up, we make the following major contributions in this paper.

- We introduce and formalize a new trajectory query based on trajectory segments by which it is possible to find trajectories that meet with the user expectation.

- We develop a trajectory segments search algorithm to seek combinations of trajectory segments and prune search space.

- We evaluate our approach based on a real dataset and a synthetic dataset. The experimental results demonstrate the effectiveness and efficiency of our approach.

The remainder of this paper is organized as follows. We formally define necessary concepts and our problem in section 2. Section 3 introduces the hybrid index for this problem. Proposed search algorithm are discussed in section 4. Experimental results are demonstrated in section 5. Finally, we have a brief review of related work in section 6 and conclude this paper in section 7 .
Table 1: Summary of Notations

\begin{tabular}{|l|l|}
\hline Notation & Definition \\
\hline $\operatorname{Tr}$ & a trajectory \\
\hline$A P S$ & $\begin{array}{l}\text { a trajectory segment of } \mathrm{Tr} \\
\text { from } p \text { to } q\end{array}$ \\
\hline$S(T r)$ & an activity point sequence \\
\hline$p$ & $\begin{array}{l}\text { a region sequence which } \mathrm{Tr} \\
\text { passes through }\end{array}$ \\
\hline$p . \varphi$ & a trajectory point \\
\hline$\alpha$ & $\begin{array}{l}\text { an activity keyword set of the } \\
\text { point }\end{array}$ \\
\hline$q$ & an activity keyword \\
\hline$Q$ & a query point \\
\hline$d(p, q)$ & a query point set \\
\hline$A P S . O M(Q)$ & $\begin{array}{l}\text { the spatial distance from } p \text { to } \\
q\end{array}$ \\
\hline $\operatorname{Tr} . B O M(Q)$ & $\begin{array}{l}\text { an ordered match from } A P S \\
\text { to } Q\end{array}$ \\
\hline$D_{\text {om }}(Q, A P S . O M(Q))$ & $\begin{array}{l}\text { the best ordered match from } \\
\text { Tr to } Q\end{array}$ \\
\hline$D_{b o m}(Q, T r . B O M(Q))$ & $\begin{array}{l}\text { the ordered match distance } \\
\text { from } A P S \text { to } Q\end{array}$ \\
\hline & tance from $\operatorname{Tr}$ to $Q$ \\
\hline
\end{tabular}

\section{PROBLEM STATEMENT}

In this section, we provide necessary definitions and give the format statement about the trajectory query problem. Table 1 summarizes notations used in this paper.

Definition 2.1 (Trajectory). A trajectory is defined as a trajectory point sequence, i.e., $\operatorname{Tr}=\left\langle p_{1}, p_{2}, p_{3} \ldots p_{n}\right\rangle$. Each trajectory point contains spatial information and temporal information.

Definition 2.2 (Trajectory Segment). Given a trajectory $\operatorname{Tr}=\left\langle p_{1}, p_{2} \ldots p_{n}\right\rangle$, a trajectory segment is a subsequence of $\operatorname{Tr}$ denoted as $\operatorname{Tr}\left\langle p_{i}, p_{j}\right\rangle=\left\langle p_{i}, p_{i+1} \ldots p_{j}\right\rangle$, where $1 \leq i \leq$ $j \leq n$.

Definition 2.3 (Activity Point Sequence). An activity point sequence is a subsequence of a trajectory $\operatorname{Tr}=\left\langle p_{1}, p_{2} \ldots p_{n}\right\rangle$, denoted as $A P S=\left\langle p_{i} \ldots p_{j}\right\rangle$, where $1 \leq i \leq j \leq n$. And each point in $A P S$ is an activity point. Activity point is a trajectory point where the point is associated with activity keywords. We use a vocabulary list to describe what people do at these places.

Definition 2.4 (Match Point). Given a query point $q$ and an activity point $p, p$ is a match point to $q$ if $p$ contains the activity keyword in $q$, i.e., $q . \alpha \in p . \varphi$. The spatial distance from $p$ to $q$ is denoted as $d(p, q)$.

Definition 2.5 (Ordered Match). Given a sequence of query points $Q$ and an activity point sequence $A P S$ in $T r$, we say $A P S$ is an ordered match to $Q$, denoted as APS.OM(Q), 
if $A P S$ has a match point set $P_{i}$ to each query point $q_{i}$. And for any pair of query points $q_{i}, q_{j}(\mathrm{i} \leq \mathrm{j})$, the index of any match point in $P_{i}$ is smaller than the index of any match point in $P_{j}$. The sum of distances from each query point to the match point set in $A P S$ is called the ordered match distance, denoted as, $D_{o m}(Q, A P S . O M(Q))$.

$$
\begin{gathered}
D\left(q_{i}, P_{i}\right)=\sum_{p_{i} \in P_{i}} d\left(q_{i}, p_{i}\right) \\
D_{\text {om }}(Q, A P S . O M(Q))=\sum_{q_{i} \in Q} D\left(q_{i}, P_{i}\right)
\end{gathered}
$$

Where $P_{i}$ is the match point set to $q_{i}$.

Definition 2.6 (Best Ordered Match). Given a collection of query points $Q$ and a trajectory $T r$, an ordered match $A P S . O M(Q)$ in $T r$ is the best ordered match, denoted as $\operatorname{Tr} . B O M(Q)$, if for any other ordered match $A P S^{\prime} . O M(Q)$ in $\operatorname{Tr}$ we have $D_{\text {om }}(Q, A P S . O M(Q)) \leq D_{\text {om }}\left(Q, A P S^{\prime} . O M(Q)\right)$. The ordered match distance of the best ordered match is called the best ordered match distance, denoted as $D_{\text {bom }}(Q, \operatorname{Tr} . B O M(Q))$.

Problem Statement. Given a sequence of query points $Q$ and a trajectory dataset $D$, the Trajectory Query Based on Trajectory Segments with Activities (TQTSA) will return $k$ trajectories from $D$ which have the smallest best ordered match distance to the query $Q$. Returned trajectories are raw trajectories or combinations of trajectory segments.

\section{INDEX STRUCTURE}

To solve TQTSA, we need find activity points which are close to query locations. Thus we can adopt a spatial index for the search of close-by trajectories for each query location separately. We adopt an efficient index called GAT [17] for the search of closest activity points. GAT index a $d$-Grid by dividing the entire query region into $2^{d} \times 2^{d}$ quad grids. Then it further builds $(d-1)$-Grid, $(d-2)$-Grid, 1-Grid, which will form a hierarchy of grids. For each bottom grid, there is a index for trajectories.

The GAT can find trajectories near a specified location efficiently, but it is not very suitable for searching matched activity points. Considering that, we construct a index for activity points to replace the original index for trajectories and organize activity points on trajectories into this index, as shown in Figure 2. With the help of this index, we can easily find activity points matched to a query point and split trajectories quickly.

Activity Point Sequence Table(APST). Considering that the most information of a trajectory are contained in activity points, we extract a complete activity point sequence of a trajectory to represent it. Then we organize all activity point sequences into a table. The purpose of this index is to quickly retrieve all activity points of a trajectory and compute the best ordered match distance from query locations to it.

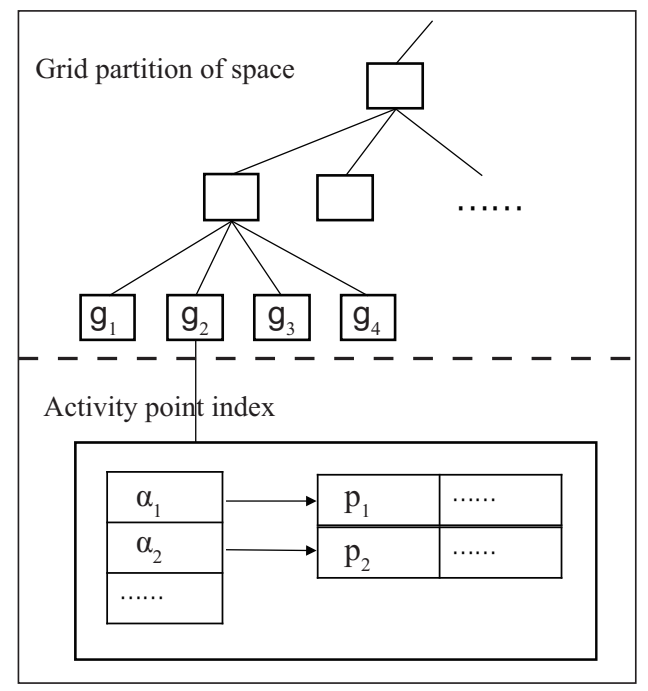

Figure 2: Hybrid index

Point Mapping Table(PMT). We also build a table to store mapping relationships between activity points and activity point sequences of trajectories. For each activity point, there is a link to the corresponding activity point sequence in the Point Mapping Table. The advantage of this table is that it can easily find the sequence which contains the specific activity point without traversing the entire activity point sequence table.

Trajectory Region Table (TRT). It is easily to determine whether two trajectories have spatial link by observing regions which two trajectories pass through. To find shared regions among trajectories efficiently, we build a table to organize region sequence for each trajectory into it. With help of TRT, we can easily discover duplicate regions between two region sequence to validate the spatial link of two trajectories. The advantage of this index will be described in detail in section 4.2 .

\section{PROPOSED SEARCH ALGORITHM}

This section introduces our search algorithm for TQTSA, called Order-sensitive Activity Trajectory Segments Search (OATSS). The basic structure of the algorithm is illustrated by Algorithm 1. In the search algorithm, we take advantage of the observation that there are a lot of trajectories that close to a part of query locations. We consider that only these trajectories are worth splitting and recombination.

Firstly, we retrieve a set of activity points near query locations within a pre-specified spatial region. Then we repeat searching activity point sequences in PMT for each pair of adjacent query locations. We retrieve candidate sequences which order-sensitive matched to a part of query locations and validate spatial links among them. When we find two activity point sequences fulfilling requirements, we extract qualified a part of them and combine them into a new activity point sequence according to combination rules. After 
combination process, we insert the new sequence into a result set. After processing all query locations, we compute the best ordered match distance of sequences in the result set and select top- $k$ as results from the result set. Finally, we convert the top- $k$ activity point sequences to complete trajectories and return them to users.

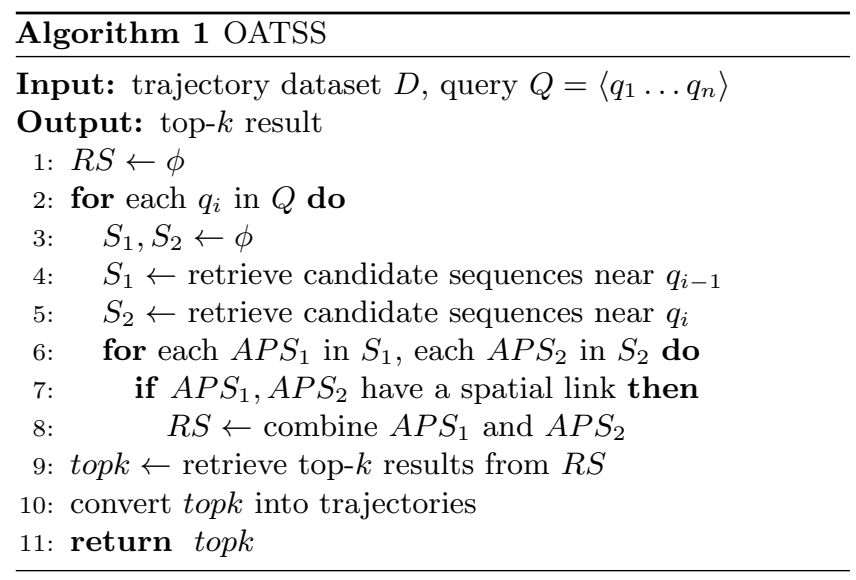

\subsection{Candidate Retrieval}

A candidate activity point sequence is possible to become a part of results for the query. Since trajectory can consist of several places spanning a large area, we can obtain a set of candidates which are close to at least one of query locations and contain the activity keyword at that location. Considering that trajectories which far from query locations are valueless and slow down the running process, we retrieve trajectories within a pre-specified distance limit $\delta_{d}$, such as $10 \mathrm{~km}$.

Given a pair of adjacent query location $q_{i}, q_{i+1}$, the retrieval process starts from searching candidate activity point sequences which are close to query locations. For $q_{i}$, from GAT, we retrieve grids near $q_{i}$ within a distance limit and obtain activity points matched to $q_{i}$ from grids. Then, we get activity point sequences contained activity points matched to $q_{i}$ and validate them whether they are qualified. For example, given an activity point sequence $A P S$ of a trajectory $T r$ and an activity activity $P$ matched to $q_{i}$, we check activity keywords contained in the trajectory segment $\operatorname{Tr}\langle$ head, $P\rangle$, where head is the head point of $T r$. If the trajectory segmen$\mathrm{t}$ is an ordered match to query location sequence $\left\langle q_{1} \ldots q_{i}\right\rangle$, $A P S$ is a candidate activity point sequence and is inserted into a candidate set $S_{1}$ (line 4 ).

As for $q_{i+1}$, we still retrieve grids near $q_{i+1}$ and obtain activity points matched to $q_{i+1}$ from grids. But in validation process, it is different. Given an activity point sequence $A P S^{\prime}$ of a trajectory $T r^{\prime}$ and a activity activity $P^{\prime}$ matched to $q_{i+1}$, we check activity keywords contained in the trajectory segment $\operatorname{Tr}^{\prime}\left\langle P^{\prime}\right.$, tail $\rangle$, where tail is the tail point of $T r^{\prime}$. If it is an ordered match to query location sequence $\left\langle q_{i+1} \ldots q_{n}\right\rangle$, $A P S^{\prime}$ is a candidate sequence and is inserted into a candidate set $S_{2}$ (line 5 ).
After filtering out candidate sequences of trajectories, we just only need to find spatial links between trajectories in $S_{1}$ and $S_{2}$.

\subsection{Validating Spatial link}

This step validates two trajectories whether they have a spatial link. On a plane, we can determine two lines whether they intersect each other through an intersection point. However it is not applicable to trajectories because trajectories are discrete. We only can validate two trajectories whether they converge on a shared region. If the shared region is small enough, we think that there is a spatial link between two trajectories. Therefore, we divide the entire query region into small cells with $1 \mathrm{~km} \times 1 \mathrm{~km}$.

Summing up the above, two trajectories have a spatial link if they have at least one of shared cells. A straightforward approach is comparing cells which two trajectories pass through one by one. But it is too loose to be useful in practice. We introduce an efficient method to validate spatial link, shown in Algorithm 2.

Given two trajectories $\operatorname{Tr}_{1}, T r_{2}$, from TRT, we firstly retrieve cells which two trajectories pass through. Here we maintain a hash table to keep track of a cell set which are visited. The key of the hash table is the index number of a cell and the value is a boolean value which represents the status of a cell. At first, we retrieve cells which $T r_{1}$ passes through. For each cell in set, we set the value is true with respect to this key. Then, we retrieve cells which $T r_{2}$ passes through. For each cell in set, we validate the value respect to the key of the cell. If there is a true value, $T r_{1}$ have a spatial link with $\mathrm{Tr}_{2}$. The algorithm also returns shared cells which help us to combine $T r_{1}$ and $T r_{2}$ into a new trajectory.

In algorithm 1 (line 7 ), given two activity point sequences of two trajectories $\operatorname{Tr}_{1}, \operatorname{Tr}_{2}$ from $S_{1}, S_{2}$, we just need validate the spatial link of trajectory segments $\operatorname{Tr}_{1}\langle P$, tail $\rangle$, $\operatorname{Tr}_{2}\left\langle\right.$ head, $\left.P^{\prime}\right\rangle$, where $P, P^{\prime}$ is match point to $q_{i-1}, q_{i}$.

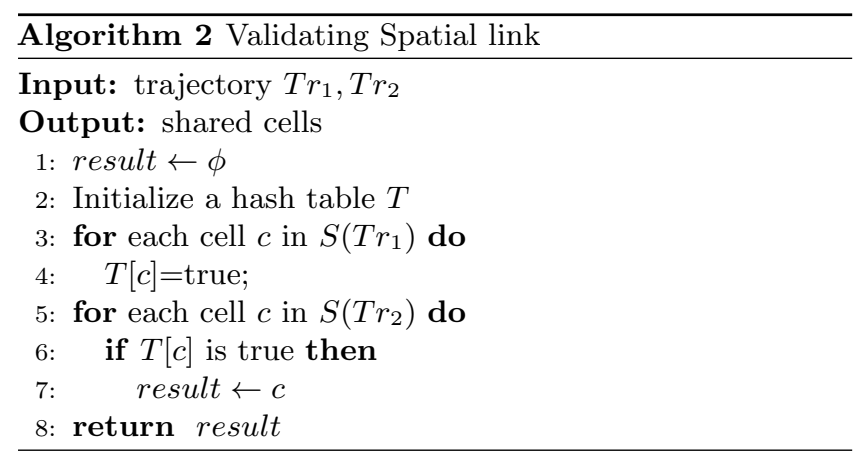

\subsection{Trajectory Combination}

The last step introduces how to combine two trajectories into a new trajectory. Since user activities start from the head of the trajectory and end at the tail of the trajectory, the split and combination process should follow the user activity sequence. 


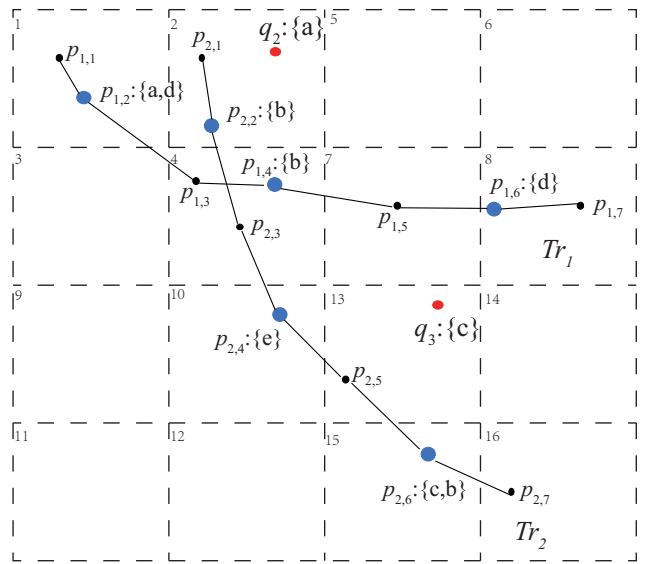

Figure 3: Trajectory combination example

As shown in Figure 3, there are two candidate trajectories $T r_{1}, T r_{2}$, where $p_{1,2}$ matched to $q_{2}$ and $p_{2,6}$ matched to $q_{3}$. In general, we need retain activity points $p_{1,2}$ and $p_{2,6}$. Thus we validate whether there is a spatial link between trajectory segment $\operatorname{Tr}_{1}\left\langle p_{1,2}\right.$, tail $\rangle$ and $T r_{2}\left\langle\right.$ head, $\left.p_{2,6}\right\rangle$. Obviously, they meet each other on the shard cell 4 . The next step, we retrieve the nearest pair of trajectory points in the shared cell from intersecting trajectories, such as $p_{1,3}$ and $p_{2,3}$, where $p_{1,3}$ is from $T r_{1}$ and $p_{2,3}$ is from $T r_{2}$. Finally we extract trajectory segments $\operatorname{Tr}_{1}\left\langle p_{1,1}, p_{1,3}\right\rangle, T r_{2}\left\langle p_{2,3}, p_{2,7}\right\rangle$ and connect $p_{1,3}$ to $p_{2,3}$ to get a new trajectory $T r^{\prime}=$ $\operatorname{Tr}_{1}\left\langle p_{1,1}, p_{1,3}\right\rangle+\operatorname{Tr}_{2}\left\langle p_{2,3}, p_{2,7}\right\rangle$. Since there may be more than one shared cell, we retrieve the one which is the nearest to the pair of query locations.

In algorithm 1, after validating the spatial link of two candidate sequences, we combine two activity point sequences and record the shared cell. After filtering out the top- $k$ results, we convert activity point sequences to complete trajectories according to the above-mentioned method.

\section{EXPERIMENTS}

\subsection{Experiments Setting}

5.1.1 Dataset. The real trajectory dateset is from the Microsoft GeoLife Project [18-20]. This dataset contains 17,621 trajectories which record a wide range of 182 users outdoor activities, such as shopping, dining, sightseeing. To get activity trajectories, we firstly pick out raw trajectories in Beijing and detect stay points [20] for each trajectory. In result, we obtain 5,371 trajectories with 18,943 stay points. For each stay point, we snap it to the closest POI as its semantic place and select the POI category as the activity keyword. The POI dataset contains 68,400 points in Beijing and covers 10 most common types of facilities and services, as listed in Table 3. All POIs are in a region whose longitude is from 115.4 to 117.6 and latitude is from 39.4 to 41.4. Since most of these POIs can be found in the database of map.baidu.com ${ }^{1}$.

\footnotetext{
${ }^{1}$ http://map.baidu.com
}

Table 2: Default Settings of Experiments

\begin{tabular}{|c|c|}
\hline Parameter & Default value \\
\hline result $k$ & 10 \\
\hline query points $|Q|$ & 3 \\
\hline the diameter of query $\delta(Q)$ & $5 \mathrm{~km}$ \\
\hline distance limit $\delta(d)$ & $10 \mathrm{~km}$ \\
\hline
\end{tabular}

The synthetic dataset is generated randomly on POI dataset. For each raw trajectory, we randomly generate a starting point, a destination and randomly generate a route connect two points. Then we sampling some points by a certain interval on the route and replace them to other random POIs near the point.

The most important difference between synthetic and real data is trajectories distribution. Trajectories in synthetic data are evenly distributed on the entire query region. But trajectories in real dataset gather in some special places such as Zhongguancun and Beijing Capital International Airport.

5.1.2 Algorithms. No baseline method exists using segment combination to search activity trajectories. Since there are methods searching activity trajectories without segment combination, we adopt one of them as a comparison, namely Order-sensitive Activity Trajectory Similarity Query (OATSQ) [17].

We compare average time cost and average match distance of our proposed method OATSS and OATSQ. The average match distance is the average distance of all returned trajectories matched to query locations. In OATSS, we retrieve trajectories which are away from query locations within $10 \mathrm{~km}$. The default values for parameters are summarized in Table 2 . In the experiment, we will vary these values to investigate the effect of each parameter. Besides, we do not change $\delta d$ because it is used in OATSS only.

For each set of experiment, we generate 30 queries and report the average running time and average match distance. Each query is generated by randomly choosing a trajectory from the dataset, and then selecting the desired number of locations and activities.

In addition, all algorithms are implemented in Java and run on a PC with Intel i7-6700k 4GHz CPU and 8GB memory.

\subsection{Performance Evaluation}

5.2.1 Effect of $k$. In the first experiment, we study the effect of the intended number of results $k$ by plotting the average time cost and average match distance of OATSQ, OATSS on both synthetic and real datasets.

As shown in Figure 4, our proposed method can find activity trajectories closer significantly than OATSQ, though it cost more time than OATSQ. With the value of result $k$ increasing, the running time of OATSS does not increase. In contrast, OATSQ cost more time to get more results. It is 
Table 3: Categories in POI Dataset

\begin{tabular}{|c|c|}
\hline Category & Number of points \\
\hline Restaurant & 13230 \\
\hline School & 9360 \\
\hline Tourist places & 8608 \\
\hline Hospital & 7845 \\
\hline Supermarket & 18101 \\
\hline Bank & 7928 \\
\hline Airport & 11 \\
\hline Park & 969 \\
\hline Cinema & 118 \\
\hline Polices & 2230 \\
\hline
\end{tabular}

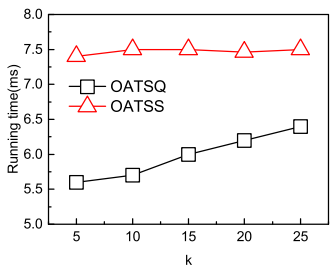

(a) synthetic data

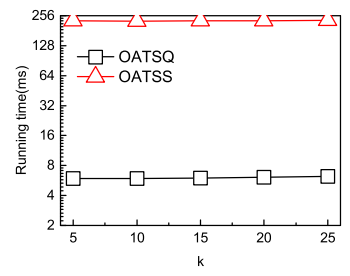

(c) real data

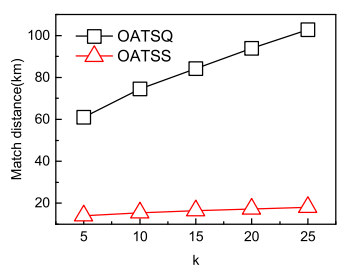

(b) synthetic data

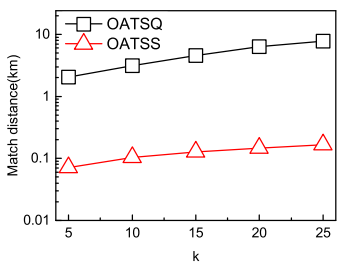

(d) real data
Figure 4: Effect of $k$

obvious that OATSQ need to expand query region and validate more trajectories when the $k$ increases. As for OATSS, the amount of returned results is influenced by the query region distance $\delta_{d}$. But the $k$ beyond the limit, OATSS cannot get more results while OATSQ may get enough required trajectories. From the Figure 4(b), 4(d), we can find that the average match distance of results on real data is smaller than on synthetic data. Besides, OATSS on real data cost more time than on synthetic data. The reason is that trajectories on real dataset gather in a small region, OATSS find more candidate trajectories on real data than on synthetic data.

5.2.2 Effect of $|Q|$. Next we study the query performance when the number of query locations $|Q|$, is varying. The results are demonstrated in Figure 5. Again, results returned by our proposed method are closer to query locations than another algorithm. As the amount of query points increases, the query time of both algorithms increase since the search space expand fast excluding OATSS on real data. Meanwhile,

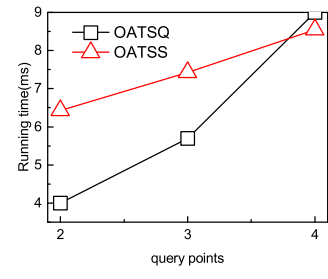

(a) synthetic data

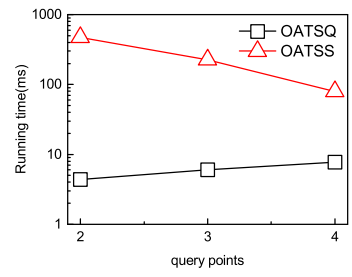

(c) real data

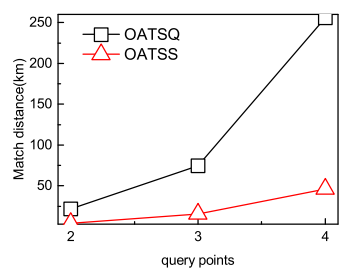

(b) synthetic data

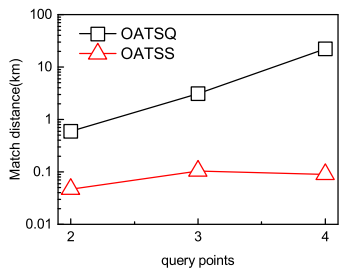

(d) real data
Figure 5: Effect of $|Q|$

in Figure 5(b), the average match distance of results returned by OATSQ increase faster significantly than OATSS. And the average match distance of OATSS increases slowly. Obviously, OATSQ need cost more time to retrieve more trajectories and validate them if qualified trajectories are far away from query locations. In addition, there are a little bit of results returned by OATSQ in this step of the experiment when the value of query points is more than 4 . But OATSS can find more qualified trajectories. Comparing the running time on both dataset, we discover that the time cost of OATSS on real dataset decreases with the amount of query points increasing. This is because that there are fewer qualified trajectories retrieved on real dataset and the search space reduces.

5.2.3 Effect of $\delta(Q)$. Then we study the effect of the distribution of query locations. The parameter $\delta(Q)$ represents the minimum distance between each pair of adjacent query locations, $q_{i}, q_{i+1}$. We choose queries with diameter varying from $5 \mathrm{~km}$ to $25 \mathrm{~km}$. Results are demonstrated in Figure 6 . In Figure 6(b) and Figure 6(d), we see that OATSS performs better than OATSQ on the match distance. On the synthetic data, with the value of $\delta(Q)$ increasing, the cost time of OATSS decreases since there are fewer candidate trajectories can be combined into new trajectories. And the average match distance of results increases. In contrast, OATSQ performs steadily. On the real data, OATSQ and OATSS performs similarly on the synthetic data.

5.2.4 Effect of Datasize. Finally we study the effect of amount of trajectories. Figure 7 illustrates the effect of the trajectory datasize and demonstrates superiority of our approach compared to conventional work. With the amount of 


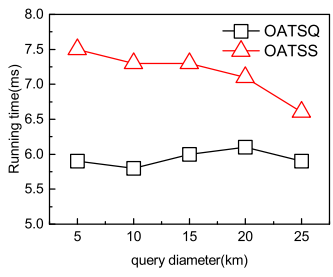

(a) synthetic data

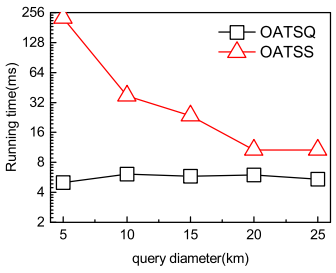

(c) real data

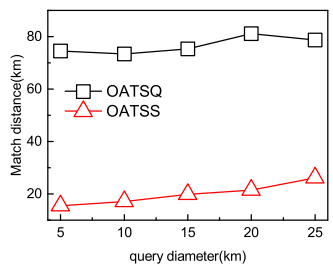

(b) synthetic data

(d) real data

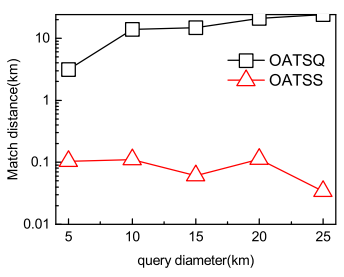

Figure 6: Effect of $\delta(Q)$

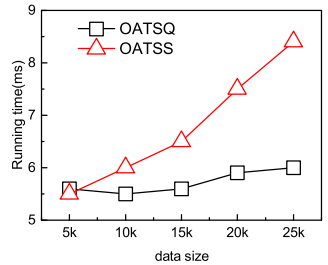

(a) synthetic data

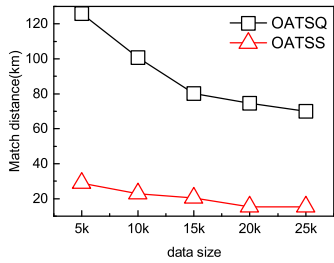

(b) synthetic data
Figure 7: Effect of datasize

trajectories increasing, the average match distance of result$\mathrm{s}$ returned by both methods decreases. Because there are more qualified trajectories close to query locations can be found. In comparison of the running time, OATSS performs worse than OATSQ. Because OATSS not only need to retrieve more candidate trajectories, but also costs much time to combine them into qualified trajectories. It is pleasing that OATSS can get superior results and the time cost is not large.

\section{RELATED WORK}

The problem of trajectory search has attracted much attention last decade. Due to the rich diversity of user requirements, there are many different algorithms to find qualified trajectories and return them to users [3, 5, 7, 8]. Initially, the work of trajectory search is constrained in spatial domain only. Given a set of locations, Chen et al. [3] proposed a method to find top- $k$ trajectories which are the closest to query locations. In addition, Dai et al. [5] considered comparing preference of speed, distance and time information in the process of trajectory search to improve user experience.

More recently, lots of work $[2,9,11,13,15,17]$ considers the fusion of spatial keywords and trajectories. In the early stage of this area, researchers studied search problem including $[4,6,10,14,16]$, which retrieve spatial objects relevant to a keyword. These work organizes objects into a spatial index and prune search region using spatial distance. Some researchers considered handling spatial keyword search on the road network. Zheng et al. [16] proposed a method to retrieve the k-nearest objects around a object on the road network. Meanwhile, many novel algorithms have been proposed to extract textual information from raw trajectories $[1,12,20]$.

Based on the textual information, many improved algorithm have been proposed to recommend meaningful trajectories to users. Shang et al. [11] proposed a method of trajectory search which aims to find trajectories associated with a specific keyword. Zheng et al. [17] studied the problem of activity trajectory search and proposed a hybrid index to solve this problem. However, it performs not very well when users provide a wrong keyword. To solve the drawback of accurate textual matching, they proposed approximate keyword search [15] to find trajectories which have similar spellings of activity keywords to the query. But these methods ignore to improve the match distance when returned trajectories are far from a part of query locations.

The research of planning route based on trajectory segments [8] is the most similar with our work. However, their work still considers spatial features without other textual information. So their techniques cannot be applied to our problem.

\section{CONCLUSION AND FUTURE WORK}

This paper discusses the problem of trajectory query based on trajectory segments with activities (TQTSA). Given a series of query locations with activity requirements, TQTSA returns the top- $k$ combinations of trajectory segments with the smallest best ordered match distance. To support the efficient trajectory segments search, we use a hybrid index to prune search space and develop a algorithm (OATSS) to solve this problem. Extensive experiments on a real dataset and a synthetic dataset demonstrate the efficiency and effectiveness of our approach.

In the future, we will consider adding influence of users' activity patterns and preferences into this system. It can recommender users preferred activity trajectories which have the same activity pattern or behavior habits as them. It is beneficial for users who have no idea about visiting the city.

\section{ACKNOWLEDGMENTS}

The authors are supported by the National Natural Science Foundation of China(No. 61572165), the Natural Science Foundation of Zhejiang Province(No. LZ15F020003). 


\section{REFERENCES}

[1] Vania Bogorny, Bart Kuijpers, and Luis Otávio Alvares. 2009 ST-DMQL: A Semantic Trajectory Data Mining Query Language. International Journal of Geographical Information Science 23, 10 (2009), 1245-1276.

[2] Wei Chen, Lei Zhao, Jiajie Xu, Kai Zheng, and Xiaofang Zhou. 2014. Ranking Based Activity Trajectory Search. In Web Information Systems Engineering - WISE 2014 - 15th International Conference, Thessaloniki, Greece, October 12-14, 2014, Proceedings, Part I. 170-185.

[3] Zaiben Chen, Heng Tao Shen, Xiaofang Zhou, Yu Zheng, and X ing Xie. 2010. Searching trajectories by locations: an efficiency study. In Proceedings of the ACM SIGMOD International Con ference on Management of Data, SIGMOD 2010, Indianapolis, Indiana, USA, June 6-10, 2010. 255-266.

[4] Gao Cong, Christian S. Jensen, and Dingming Wu. 2009. Efficient Retrieval of the Top-k Most Relevant Spatial Web Objects. PVLDB 2, 1 (2009), 337-348.

[5] Jian Dai, Bin Yang, Chenjuan Guo, and Zhiming Ding. 2015 Personalized route recommendation using big trajectory data In 31st IEEE International Conference on Data Engineering, ICDE 2015, Seoul, South Korea, April 13-17, 2015. 543-554.

[6] Ian De Felipe, Vagelis Hristidis, and Naphtali Rishe. 2008. Keyword Search on Spatial Databases. In Proceedings of the 24th International Conference on Data Engineering, ICDE 2008 , April 7-12, 2008, Cancún, México. 656-665.

[7] Elias Frentzos, Kostas Gratsias, and Yannis Theodoridis. 2007 Index-based Most Similar Trajectory Search. In Proceedings of the 23rd International Conference on Data Engineering, ICDE 2007, The Marmara Hotel, Istanbul, Turkey, April 15-20, 2007. $816-825$.

[8] Jingsheng Jiang, Chong $\mathrm{Xu}$, Jian $\mathrm{Xu}$, Ming $\mathrm{Xu}$, Ning Zheng, and Kaiwei Kong. 2016. Route planning for locations based on trajectory segments. In Proceedings of the 2nd ACM SIGSPATIAL Workshop on Smart Cities and Urban Analytics, UrbanGIS@SIGSPATIAL 2016, Burlingame, California, USA, October 31, 2016. 6:1-6:8

[9] Huiwen Liu, Jiajie Xu, Kai Zheng, Chengfei Liu, Lan Du, and Xian Wu. 2017. Semantic-aware Query Processing for Activity Trajectories. In Proceedings of the Tenth ACM International Conference on Web Search and Data Mining, WSDM 2017 Cambridge, United Kingdom, February 6-10, 2017. 283-292.

[10] Siqiang Luo, Yifeng Luo, Shuigeng Zhou, Gao Cong, and Jihong Guan. 2014. Distributed Spatial Keyword Querying on Road Networks. In Proceedings of the 17th International Conference on Extending Database Technology, EDBT 2014, Athens, Greece, March 24-28, 2014. 235-246.
[11] Shuo Shang, Ruogu Ding, Bo Yuan, Kexin Xie, Kai Zheng, and Panos Kalnis. 2012. User oriented trajectory search for trip recommendation. In 15th International Conference on Extending Database Technology, EDBT '12, Berlin, Germany, March 2730, 2012, Proceedings. 156-167.

[12] Han Su, Kai Zheng, Kai Zeng, Jiamin Huang, Shazia Wasim Sadiq, Nicholas Jing Yuan, and Xiaofang Zhou. 2015. Making sense of trajectory data: A partition-and-summarization approach. In 31st IEEE International Conference on Data Engineering, ICDE 2015, Seoul, South Korea, April 13-17, 2015. 963-974.

[13] Sheng Wang, Zhifeng Bao, J. Shane Culpepper, Timos Sellis, Mark Sanderson, and Munkh-Erdene Yadamjav. 2016. Interactive Trip Planning Using Activity Trajectories. In Proceedings of the 21st Australasian Document Computing Symposium, ADCS 2016, Caulfield, VIC, Australia, December 5-7, 2016. 77-80.

[14] Dongxiang Zhang, Chee-Yong Chan, and Kian-Lee Tan. 2014. Processing spatial keyword query as a top-k aggregation query. In The 37th International ACM SIGIR Conference on Research and Development in Information Retrieval, SIGIR '14, Gold Coast, QLD, Australia - July 06 - 11, 2014. 355-364.

[15] Bolong Zheng, Nicholas Jing Yuan, Kai Zheng, Xing Xie, Shazia Wasim Sadiq, and Xiaofang Zhou. 2015. Approximate keyword search in semantic trajectory database. In 31st IEEE International Conference on Data Engineering, ICDE 2015, Seoul, South Korea, April 13-17, 2015. 975-986.

[16] Bolong Zheng, Kai Zheng, Xiaokui Xiao, Han Su, Hongzhi Yin, Xiaofang Zhou, and Guohui Li. 2016. Keyword-aware continuous $\mathrm{kNN}$ query on road networks. In 32nd IEEE International Conference on Data Engineering, ICDE 2016, Helsinki, Finland, May 16-20, 2016. 871-882.

[17] Kai Zheng, Shuo Shang, Nicholas Jing Yuan, and Yi Yang. 2013. Towards efficient search for activity trajectories. In 29th IEEE International Conference on Data Engineering, ICDE 2013, Brisbane, Australia, April 8-12, 2013. 230-241.

[18] Yu Zheng, Quannan Li, Yukun Chen, Xing Xie, and Wei-Ying Ma. 2008. Understanding mobility based on GPS data. In UbiComp 2008: Ubiquitous Computing, 10th International Conference, UbiComp 2008, Seoul, Korea, September 21-24, 2008, Proceedings. $312-321$.

[19] Yu Zheng, Xing Xie, and Wei-Ying Ma. 2010. GeoLife: A Collaborative Social Networking Service among User, Location and Trajectory. IEEE Data Eng. Bull. 33, 2 (2010), 32-39.

[20] Yu Zheng, Lizhu Zhang, Xing Xie, and Wei-Ying Ma. 2009. Mining interesting locations and travel sequences from GPS trajectories. In Proceedings of the 18th International Conference on World Wide Web, WWW 2009, Madrid, Spain, April 20-24, 2009. 791-800. 